\title{
On the parameters influencing the deposition of polystyrene colloidal crystals
}

\author{
María Yoldi ${ }^{\mathrm{b}}$, Cristina Arcos ${ }^{\mathrm{c}}$, Bernd-R. Paulke ${ }^{\mathrm{a}}$, Rafael Sirera ${ }^{\mathrm{c}}$, \\ Wenceslao González-Viñas ${ }^{\mathrm{b}, *}$, Eckhard Görnitz ${ }^{\mathrm{a}}$ \\ ${ }^{a}$ Institute for Applied Polymer Research, Geiselbergstr. 69, D-14476 Potsdam-Golm, Germany \\ ${ }^{\mathrm{b}}$ Complex Systems Group. Department of Physics and Appl. Math., University of Navarra, Irunlarrea s/n, E-31080 Pamplona, Spain \\ ${ }^{\mathrm{c}}$ Depatment of Chemistry and Soil Sci., University of Navarra, Irunlarrea s/n, E-31080 Pamplona, Spain
}

Received 30 January 2007; received in revised form 2 April 2007; accepted 16 April 2007

Available online 25 April 2007

\begin{abstract}
Colloidal crystals of polystyrene particles of 1.0, 1.4 and $2.8 \mu \mathrm{m}$ diameter have been prepared by vertical deposition. The influence of parameters such as temperature, particle size and concentration as well as dispersion medium has been studied. The size of domain and the crystalline structure of the particle arrays have been analyzed by optical microscopy. The quality of the crystals has been improved (minimizing cracks) by controlling sedimentation (density matching), evaporation (volatility of the medium) and drying (co-solvents).
\end{abstract}

(C) 2007 Elsevier B.V. All rights reserved.

PACS: 82.70.Dd; 81.16.D

Keywords: Vertical deposition; Colloidal crystals; Cracks; Density matching; Co-solvents

\section{Introduction}

Colloidal crystals are close-packed arrays of colloidal particles, whose structures present long range order compared to the diameter of the particles. The studies on the fundamentals of colloidal crystallization [1-8] showed that, under the appropriate conditions, colloidal particles assemble spontaneously into ordered structures. When colloidal particles are partly immersed in a thin layer of liquid, lateral capillary forces cause an attractive interaction between them. Particles are pushed together and nucleate, forming a close-packed ordered monolayer. Surface stabilization of the particles (colloidal stability) is important to prevent uncontrolled aggregation in the early stage of self-assembly [9]. Then, flow of solvent brings in more spheres and the monolayer grows [10]. Thus, the forces of surface tension in the meniscus region are responsible to bring near the spheres, consolidating them into an ordered crystal. The formation of $3 \mathrm{D}$ ordered structures is more complicated than the preparation of a monolayer. In this case, fcc structure is energetically slightly more stable [11-13]. Thus, quick colloidal crystallization can not lead to this structure unless there is

\footnotetext{
* Corresponding author.

E-mail address: wens@fisica.unav.es (W. González-Viñas).
}

another mechanism which allows these preference on an out-ofequilibrium basis. This mechanism is known as convective assembly hypothesis $[14,10]$ and is based on the important role which plays the solvent flow through the interstitial sites during the opal growth. Synthetic opals usually contain numerous defects [15] (vacancies, dislocations, stacking faults and grain boundaries), which reduce their applications [16].

To improve this situation, more recent research has explored alternative approaches, improving the spontaneous self-assembly by controlled external forces. Gravitational sedimentation [17-19] or sedimentation forced by different agents as electrostatic immobilization [20], colloidal crystal templating [21], shear alignment [22], oscillatory shear [23] or flow assisted electrophoresis [24,25] and vertical deposition [26-28], which is the technique we have selected, are some examples. The efficiency of the vertical deposition $[9,29]$, relies on the balance between solvent evaporation and particle sedimentation. When sedimentation is faster than solvent evaporation, the self-organization of the particles does not take place. Consequently, the vertical deposition technique is usually successful for relatively small particles [29], but large ones sedimented too fast and it is difficult to find the narrow range of the parameters of control which allows colloidal crystallization. The rate of evaporation can be controlled by 


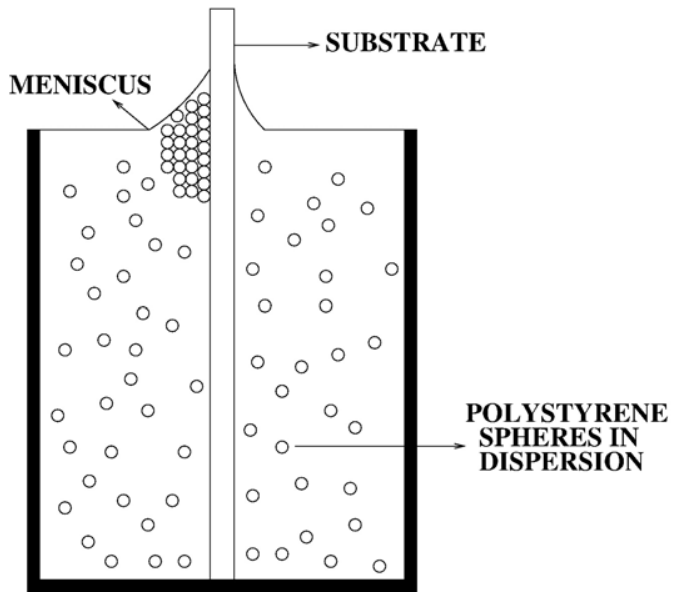

Fig. 1. Sketch of the vertical deposition procedure.

type of solvent, temperature and relative humidity [30] and the rate of sedimentation by solvent and material density (density matching), and by convective flows [31].

The aim of this work is to study the influence of some parameters on the formation of colloidal crystals in the vertical deposition process, which are temperature, particle concentration, particle size and solvent properties. Controlling evaporation (by temperature and variations of the dispersion medium), sedimentation (by density matching) and drying (by addition of co-solvents) the array quality, which was characterized by domain size analysis using optical microscopy, could be improved.

\section{Experimental section}

\subsection{Substrates and material}

Hydrophobic polystyrene (PS) latex particles, electrostatically stabilized, with a narrow size distribution and diameters of $1.0 \mu \mathrm{m}$ and $1.4 \mu \mathrm{m}$ were prepared by an emulsifier-free, aqueous radical polymerization $[32,33]$. The $1.4 \mu \mathrm{m}$ particles were coated by a hydrophilic shell by a procedure described in [34] in order to decrease van der Waals interaction between them drastically and to support position-change processes during crystallization. Large, uncharged PS particles, sterically

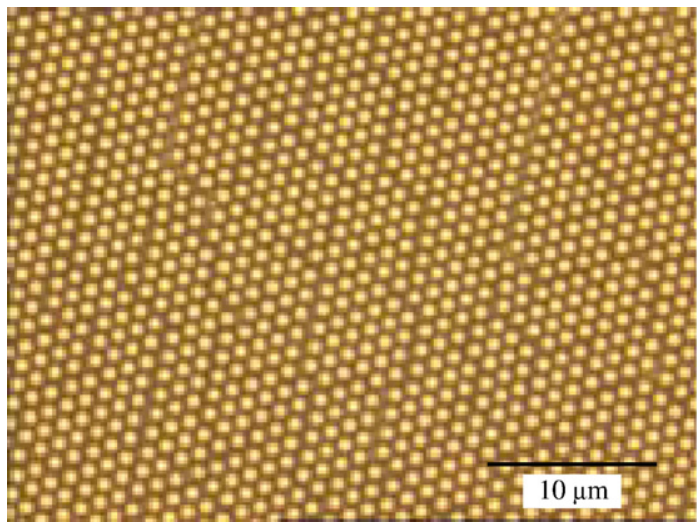

Fig. 2. Optical micrography of a sample prepared in water.

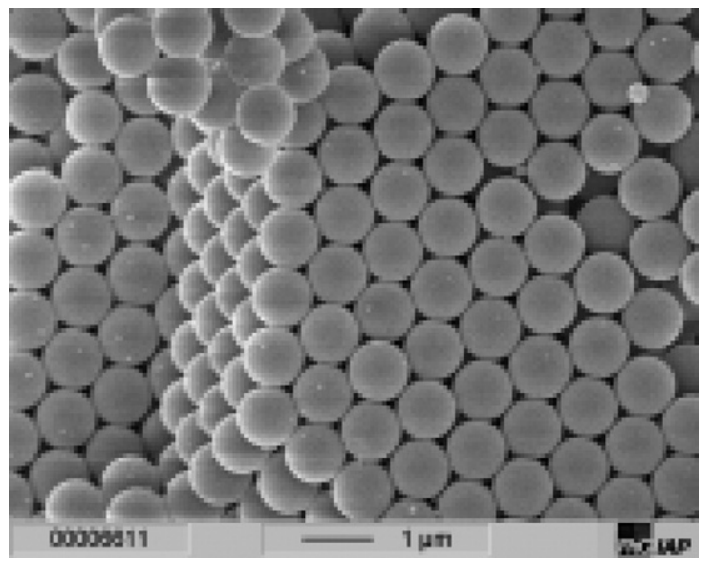

Fig. 3. S.E.M. micrograph for a fcc stacking (ABCABC).

stabilized, with a diameter of $2.8 \mu \mathrm{m}$ were prepared by nonaqueous dispersion polymerization [34,35]. Both types of latex present a polydispersity index less than 0.05 . The density of the particles is $\rho_{\text {PS }}=1.054 \mathrm{~g} / \mathrm{cm}^{3}$ and their refractive index $n=1.59$. The $\mathrm{pH}$ of the dispersions is 5.8. The surface charge density of the $1.4 \mu \mathrm{m}$ core-shell particles is $6.3 \mu \mathrm{C} / \mathrm{cm}^{2}$ and the charge of the sterically stabilized particles is $0.5 \mu \mathrm{C} / \mathrm{cm}^{2}$.

Particle size and polydispersity were determined by Dynamic Light Scattering (1.0 and $1.4 \mu \mathrm{m}$ particles) and by Fraunhofer Diffraction $(2.8 \mu \mathrm{m}$ particles). Dynamic Light Scattering experiments were performed using a Zetamaster S and Fraunhofer Diffraction with a Mastersizer X, both from Malvern Instruments. The surface charge density of the particles was determined by titration with a $0.01 \mathrm{mM}$ solution of the cationic polyelectrolyte poly(diallyldimethyl-ammonium chloride), where the point of zero charge was detected from the streaming potential by means of a Mütek PCD 03 particle charge detector.

Aqueous ammonium hydroxide (25\% in volume) was provided by PANREAC and hydrogen peroxide $30 \%$ in volume) by Merck. Pure (>99\%) ethanol, glycerol, ethylenglycol-monobutylether and N-methyl-2-pyrrolidon were provided by Aldrich. Dipropylenglycol-dimethylether (DPGDME) and dipropylen-glycolmethyletheracetate by DOW Europe $\mathrm{GmbH}$ and pure diethylenglycol-dimethylether by ACROS.

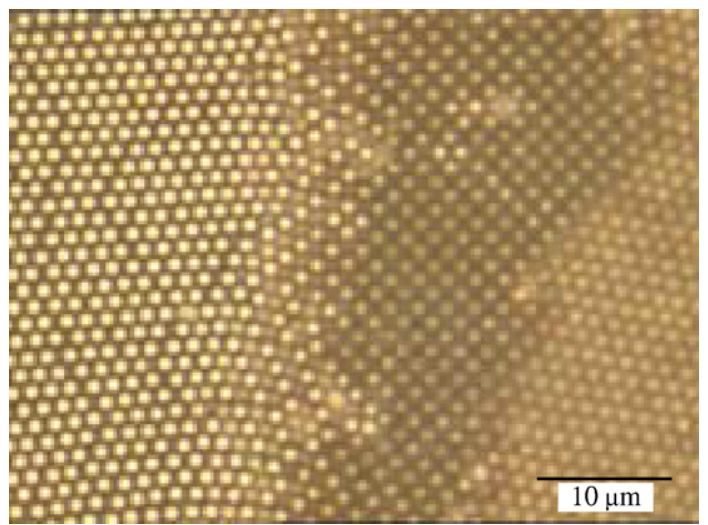

Fig. 4. Cubic transition from a monolayer to a bilayer. 


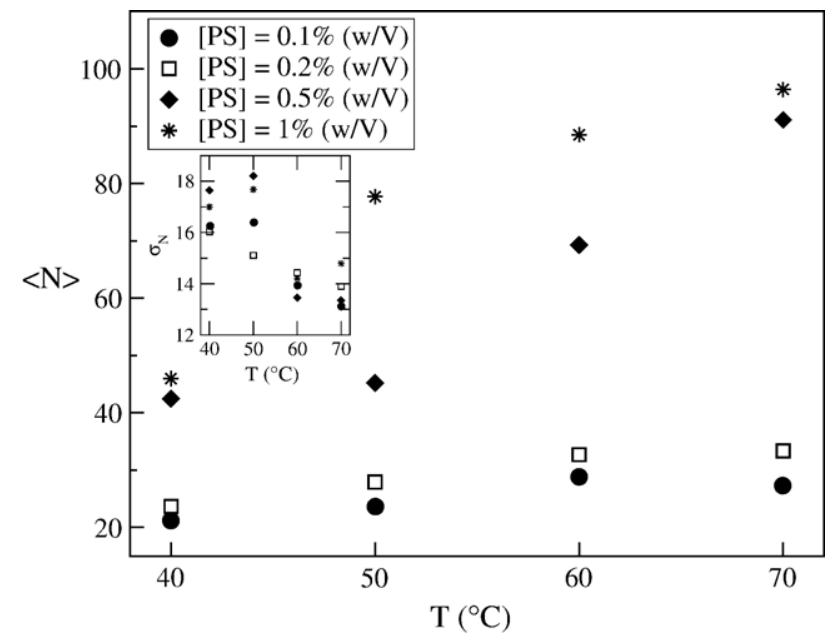

Fig. 5. Average size of domain of the top layer, $\langle N\rangle$, vs. temperature, for different concentrations of PS with a particle diameter of $1.4 \mu \mathrm{m}$. Samples prepared in water. The standard deviation is shown in the inset.

Ultra pure water, $1 \mu \mathrm{S} / \mathrm{cm}$ conductivity, was used from a Milli-Q water system, and heavy water $\left(\mathrm{D}_{2} \mathrm{O}\right)$ was filtered through $0.22 \mu \mathrm{m}$ Millipore filter.

Glass slides, provided by Roth Karlsruhe, (cut to size $12 \mathrm{~mm} \times 25 \mathrm{~mm}$, thickness $1 \mathrm{~mm}$ ) were used as substrates and pre-treated as described below.

\subsection{Experimental system and method}

The latex was first filtered using an $8 \mu \mathrm{m}$ filter and then diluted with the medium of dispersion to concentrations ranging from 0.1 to $1.0 \%$ (weight/volume). With $1.4 \mu \mathrm{m}$ particles, ultra-pure water, different ultra-pure water/ethanol mixtures, and water with small amounts of water-soluble and high-boiling co-solvents exhibiting different solvent power to PS, such as glycerol, ethylenglycol-monobutylether, N-methyl2-pyrrolidon, dipropylenglycol-dimethylether (DPGDME), dipropylenglycolmethyletheracetate and diethylen-glycoldimethylether were used as media of deposition. In the case of a fast sedimentation with $2.8 \mu \mathrm{m}$ particles, deposition from ultra-pure water and ultra-pure water/heavy-water mixtures was compared.

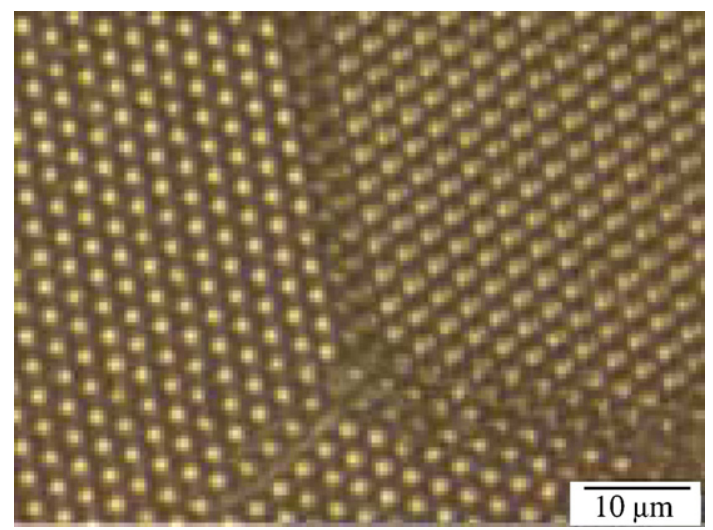

Fig. 6. Detail of a crack.

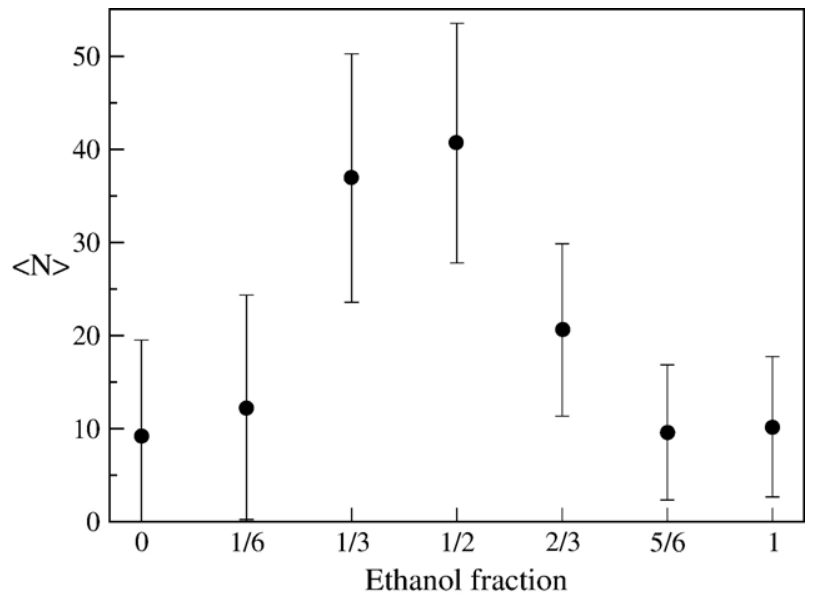

Fig. 7. Average size of domain of the top layer $\langle\mathrm{Ni}\rangle$, vs. ethanol fraction in a water-ethanol mixture. The PS particles of $1.4 \mu \mathrm{m}$ had a $0.1 \%(\mathrm{w} / \mathrm{V})$ concentration. The temperature was $70^{\circ} \mathrm{C}$.

The substrates were hydrophilized into a 1:1:5 mixture of ammonium hydroxide:hydrogen peroxide:water, for $30 \mathrm{~min}$ at $67{ }^{\circ} \mathrm{C}$.

In a representative procedure, a cylindrical plastic vial (inner diameter, ca. $13 \mathrm{~mm}$; volume, $2.5 \mathrm{~mL}$ ) was filled with approximately $1.5 \mathrm{~mL}$ of particle suspension. Then, it was sonicated again. Finally the substrate was placed into the vial, which was put on a thermobank (COESFELD Thermostair), giving the possibility to locate vials at different constant temperatures during one experiment. Usually, as the slide was slightly tilted, deposition of the particle array occurred mostly on the one side of the glass (Fig. 1).

\subsection{Characterization}

Large latex particles (diameter $>0.7 \mu \mathrm{m}$ ) can be easily visualized by optical microscopy. For this purpose, we used an OLYMPUS BX60 microscope equipped with a Sony CCD model DXC9100P and imaging analysis software analySIS 3.0. Optical micrographies of the colloidal crystals were used for the evaluation of the crystalline structure and the determination of the domain size by image analysis.

The average size of domain of the top layer $(\langle\mathrm{N}\rangle)$ of every sample was determined using the software"VIDEOKLITE”. 20 micrographs, randomly taken, were analyzed for each sample. The linear size of the top layer of the domains were measured and converted to a dimensionless magnitude using the particle diameter. From this point, this is what we will call the domain size. The average domain size is calculated by averaging the partial results from the 20 micrographs.

\section{Results and discussion}

Macroscopically, the samples were not uniform but showed the characteristic appearance in stripes, typical from the vertical deposition. The stripes reveal that the number of deposited layers varies along the direction of deposition. According to Shmuylovitch [36], the reason for these stripes could be that the 


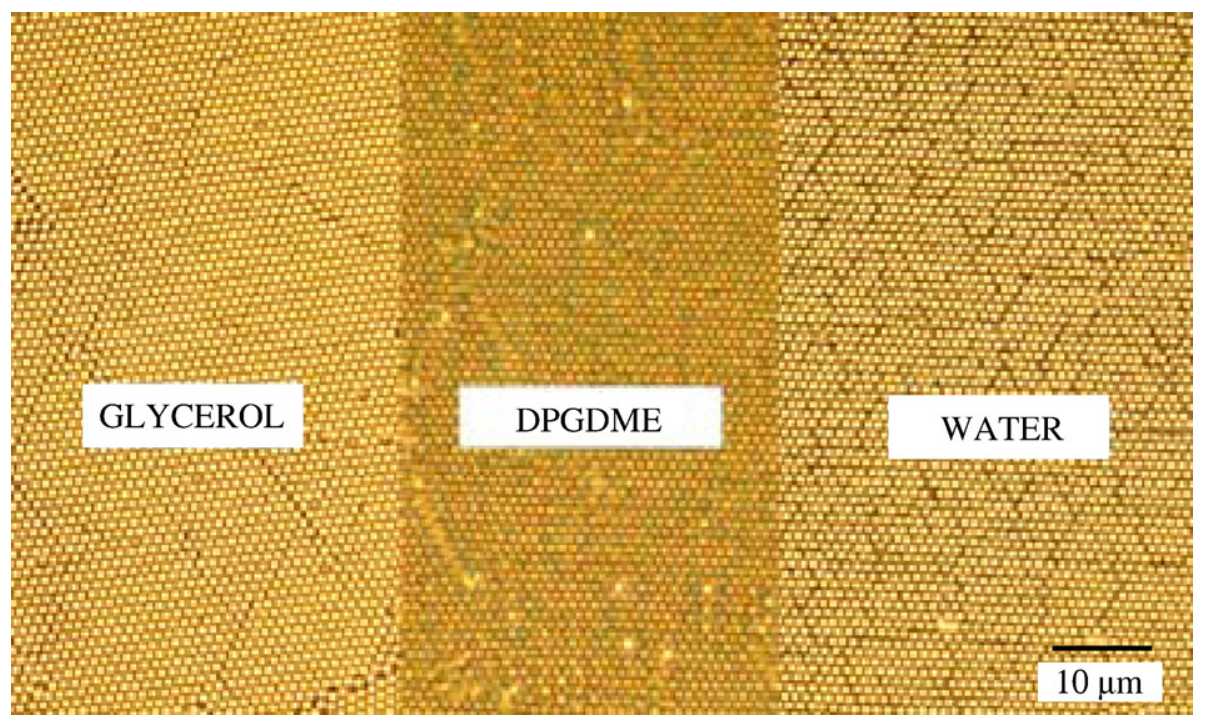

Fig. 8. Optical micrograph comparing the presence of cracks in samples prepared in water, in water with glycerol and in water with dipropylenglycol-dimethylether (DPGDME).

meniscus does not draw back with constant velocity, but discontinuously: surface tension holds the meniscus film together; when there are no particles near the film, the tension becomes too high, the film ruptures and a new meniscus starts to grow. As a result, systematic change of the transmission and reflection colours of the films due to Bragg diffraction can be observed.

Optical images of the top view of these samples show that the colloids are arranged in a close-packed arrangement, where each sphere touches six others in one layer. This hexagonally close packed arrangement is well-known in colloidal crystals, because it is the optimal geometry to minimize the repulsive interactions between particles (Fig. 2).

When the third dimension is considered, three different stackings are relevant: $\mathrm{ABCABC} .$. (face centered cubic, fcc), ABABAB... (hexagonal close-packing, hcp) or ABCBA... (random hexagonally close-packing, rhcp). The diverse colours found in optical transmission microscopy at low magnification show, that even for the same number of layers different structures exist, but it is not simple to distinguish among them.

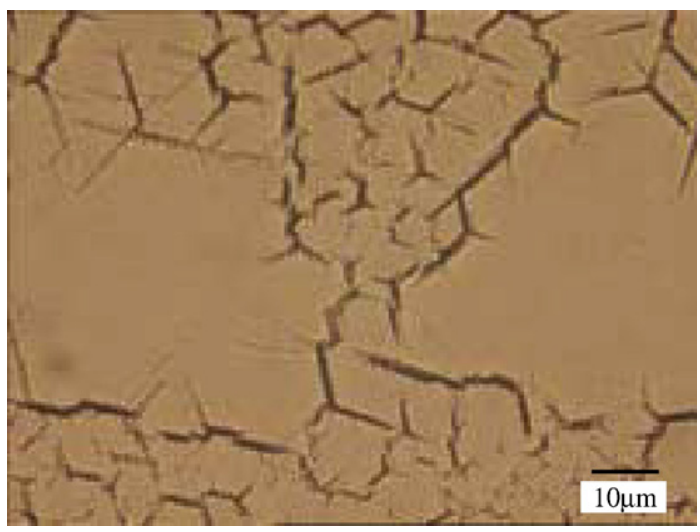

Fig. 9. Optical micrograph of sample prepared in water with N-methyl-2pyrrolidon.
Traditionally [37], S.E.M. has been used to determine the 3D structure (Fig. 3).

The arrays were hexagonal, except from the transition areas from one to two layers (or from two to three), where cubic arrays were observed. In the transition areas, the number of layers could be regarded as fractional $(1.5,2.5, \ldots)$. While the hexagonal arrangement is the most stable for a packing of spheres in "complete layers", cubical arrays seem to be the most stable for "fractional layers". According to Lazarov [38] and Leiderer group [39,40], cubic arrays take place when colloidal structure goes from one to two layers (Fig. 4). Also, a recent paper of Norris' group is devoted to this kind of thickness transitions in convective assembly [10].

It is observed that the average size of domain increases with particle concentration and also with temperature (Fig. 5). Temperature mainly affects the evaporation rate of the solvent. In this way, the contact line is swept out more quickly and particle sedimentation does not occur significantly during the deposition. Moreover, if the temperature is increased,

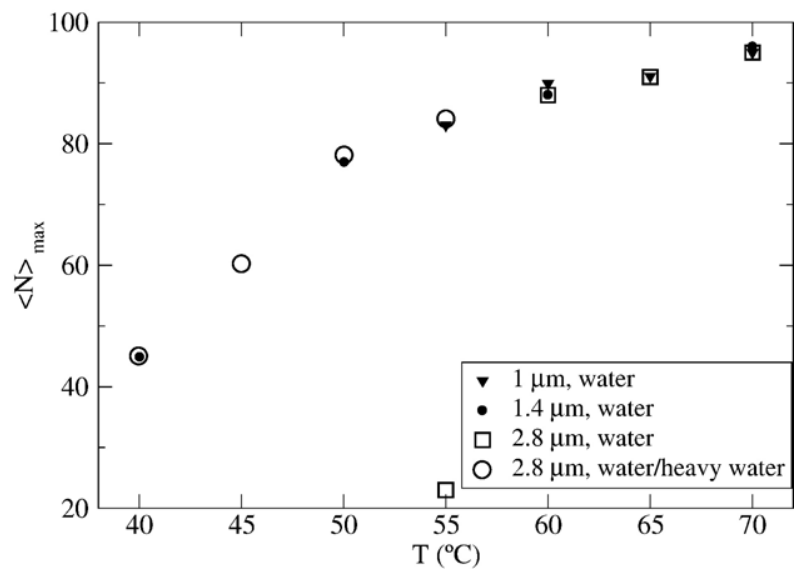

Fig. 10. Maximum average size of domains of the top layer $\langle\mathrm{N}\rangle_{\max }$, vs. temperature for particles of different size. 
sedimentation is partially reduced due to thermally enhanced advective flows in the bulk and in the surface, which also increases order in the sample by stirring up particles locally and letting them more time to achieve the energetically favored site. Because of that, in some temperature region an increase of domain size with temperature was found.

In spite of this, different types of defects can appear in the samples. Neither the number of sphere vacancies nor the number of dislocations were significant. However, cracks, which are planar defects perpendicular to the glass substrate (Fig. 6), affect strongly the domain size.

In order to increase the rate of evaporation even more, we also did the experiments mixing the previous dispersion medium (water) with a more volatile liquid (ethanol).

The results of the experiments developed in aqueousethanolic medium showed that the average domain size increases with the fraction of ethanol in volume until 0.5 , from which sedimentation due to the increasing density difference between particle and dispersion medium becomes predominant and self-organization abates (Fig. 7).

Petukhov [41] argued that the capillary forces generated during the drying process destroy the long-range order and break the crystal into smaller crystallites with slightly different orientations. According to them, the number of cracks should be reduced by either controlling or even avoiding the drying stage.

In order to modify the drying process, crystallization also was made in water with different water-miscible co-solvents, which act more or less as swelling agents for the particle material (PS). Glycerol and DPGDME (dipropylenglycoldimethylether) were revealed to be the best co-solvents for our purpose. The size of domain is up to 5 times bigger by using either glycerol or DPGDME, because the number of cracks formed during the drying stage decrease (Fig. 8). As the cosolvents are not volatile at room temperature, they remain between the ordered particles in the colloidal crystal, slowing down the drying process or even avoiding the drying stage completely. On one hand, as the colloidal crystals remain wet, no cracks are formed. It is important to notice that only 0.5 to $1.0 \%$ (weight/volume) of co-solvent is used, in order to achieve stable close-packed arrays with only a limited particle swelling. On the other hand, co-solvents change the effective refraction index of the colloidal crystal. In fact, wet colloidal crystals are more transparent than dry ones. Glycerol resulted in the largest increase of transparency.

The particle swelling in the other co-solvents is stronger; the glass transition temperature $\left(T_{\mathrm{g}}\right)$ of the PS decreases due to the plastification, and coalescence (film formation) takes place. Because of that, these co-solvents do not only reduce the number of cracks, but the particulate structure of the crystals gradually disappears (Fig. 9).

A rough classification of the co-solvents can be made by their total Hilde brand solubility parameters $\delta_{\mathrm{t}}$ [42]. Ethylenglycolmonobutylether $\left(\delta_{t}=20.8 \mathrm{MPa}^{1 / 2}\right)$, N-methyl-2-pyrrolidon $\left(\delta_{t}=23.0 \mathrm{MPa}^{1 / 2}\right)$ and dipropylenglycol-methyletheracetate $\left(\delta_{t}=19.3 \mathrm{MPa}^{1 / 2}\right)$ have solubility parameters close to that of PS $\left(\delta_{t}=21.3 \mathrm{MPa}^{1 / 2}\right)$ qualifying them as "good" solvents for the polymer. On the other hand, the values for glycerol $\left(\delta_{t}=36.1 \mathrm{MPa}^{1 / 2}\right)$ and DPGDME $\left(\delta_{t}=15.5 \mathrm{MPa}^{1 / 2}\right)$ are outside of the solubility window of PS. With this "co-solvents" obviously only a slight surface swelling of the PS-particles takes place which is sufficient for the reduction of crack formation.

From this point, we would like to compare the size of domain between colloidal crystals made with particles of different diameters $(1.0,1.4$ and $2.8 \mu \mathrm{m})$. For that, firstly we considered the averages of domain size corresponding to several different conditions (temperature, particle diameter and concentration) of samples prepared in water. Then, we took for each temperature and particle diameter, the maximum average size $\langle\mathrm{N}\rangle_{\max }$ and we plotted it in Fig. 10 vs. temperature. In this sense, $\langle\mathrm{N}\rangle_{\max }$, could be regarded as the average domain size under optimal particle concentration.

In Fig. 10, it can be seen that the maximum average dimensionless domain size, $\langle\mathrm{N}\rangle_{\max }$, does not depend on the particle size (at least for 1 and $1.4 \mu \mathrm{m}$ ). It is very interesting to focus on the results obtained at $55^{\circ} \mathrm{C}$. At this temperature, the domain size of the colloidal crystals prepared with $2.8 \mu \mathrm{m}$ particles is appreciably smaller than the domain size of the crystals made with 1 and $1.4 \mu \mathrm{m}$ ones. However, if a mixture water/heavy water $(50 \%$ in volume) was used as dispersion medium, the domain size of these colloidal crystals prepared with $2.8 \mu \mathrm{m}$ particles increased up to the expected value (these points also were added to Fig. 10). The results showed that for small particles, temperature is not so critical, but for large particles high temperature is required in order to prevent sedimentation. Other possibility is to increase the density of the dispersion medium in order to diminish sedimentation and to let the particles time enough to organize themselves into colloidal crystals.

\section{Conclusions}

Stabilization against early aggregation is one of the major preconditions for self assembly of colloidal particles into crystalline ordered structures. This is in particular true for large particles (bigger than $1 \mu \mathrm{m}$ diameter), which present problems in vertical deposition due to their inertia against thermal (Brownian) movement and due to their high rate of sedimentation. We chose hydrophilic particle shells to avoid this uncontrolled aggregation during the deposition process. In this way, colloidal crystals up to $2 \mathrm{~cm}^{2}$ in size with up to 50 layers and monocrystalline domains of up to 100 particle diameters in size (corresponding to around $10^{4}$ particles on the top layer of the domain) were prepared in this work. This might be of importance to optical applications. The domains present preferably stackings of hexagonally close packed particle layers resulting in fcc, hcp and rhcp crystalline structures.

The experiments showed that the size of the monocrystalline domains can be improved by optimizing the main parameters of control, which are temperature, particle size and concentration as well as the properties of the dispersion medium (as influenced by co-solvents). Although this optimization depends on the size and nature of the colloidal particles, once the system is optimized to get the maximum average size of domains, this 
maximum non-dimensional size $\langle\mathrm{N}\rangle_{\max }$ does not depend on particle size.

Increased thermal mobility of the particles, which was achieved by high temperature (from 40 to $70{ }^{\circ} \mathrm{C}$ ), resulted in improved order of the deposited structures. The domain size can be balanced by the particle concentration (from 0.1 to $1 \%$ weight/volume) and the evaporation rate. Regarding the influence of the dispersion medium, an increased evaporation rate, which could be achieved by adding up to 0.5 fraction in volume of ethanol, significantly improved the array quality. Moreover, density matching is another of the relevant parameters as can be seen in higher fractions of ethanol and in experiments with largest particles $(2.8 \mu \mathrm{m})$ where the density matching could be obtained by mixing water and heavy water. In this case, the high density of the medium prevented sedimentation, at the deposition time scales, and thus allowing assembly of the particles.

Dislocations and cracks, which are formed during drying stage after particle deposition, are the most obvious types of defects in colloidal crystals. The number of these defects can be dramatically reduced by adding small amounts of a co-solvent to the dispersion medium. In this way, a slight plastification of the particle surfaces takes place and complete drying of the particle film is avoided. Most advantageously in the selfassembly of polystyrene colloidal particles are, among the investigated additives, glycerol and dipropylenglycol-dimethylether in the concentration range between 0.5 and $1 \%$ in volume. By adding these compounds to the dispersion medium, the size of domains is increased up to 5 times.

\section{Acknowledgements}

We acknowledge to Dr. J. Wagner for fruitful discussions. We are also indebted for technical support in polymerization to K. Schauer and to Dr. M. Pinnow for S.E.M.. MY acknowledges MECD scholarship. This work was partly supported by the Spanish MCyT (projects BFM2002-02011 and MAT200302369), and PIUNA.

\section{References}

[1] P. Pieranski, Contemp. Phys. 24 (1983) 25.

[2] R.J. Carlson, S.A. Asher, Appl. Spectrosc. 38 (1984) 297.

[3] P.N. Pusey, W. van Megan, Nature 320 (1986) 340.

[4] P.N. Pusey, W. van Megan, P. Bartlett, B.J. Ackerson, J.G. Rarity, S.M. Underwood, Phys. Rev. Lett. 63 (1989) 2753.

[5] K.E. Davis, W.B. Russel, W.J. Glantschnig, Science 245 (1989) 507.

[6] A.D. Dinsmore, J.C. Crocker, A.G. Yodh, Curr. Opin. Colloid Interface Sci. 3 (1998) 5.

[7] A.P. Gast, W.B. Russel, Phys. Today (1998) 24.

[8] D.G. Grier, MRS Bull 23 (1998) 21.
[9] A.S. Dimitrov, K. Nagayama, Langmuir 12 (1996) 1303.

[10] L. Meng, H. Wei, A. Nagel, B.J. Wiley, L.E. Scriven, D.J. Norris, Nano Lett. 6 (2006) 2249.

[11] L.V. Woodcock, Nature 385 (1997) 141.

[12] P.G. Bolhuis, D. Frenkel, S.-C. Mau, D.A. Huse, Nature 388 (1997) 236.

[13] S.-C. Mau, D.A. Huse, Phys. Rev., E Stat. Phys. Plasmas Fluids Relat. Interdiscip. Topics 59 (1999) 4396.

[14] D.J. Norris, E.G. Arlinghaus, L. Meng, L. Heiny, L.E. Scriven, Adv. Materials 16 (2004) 1393

[15] Y.A. Vlasov, V.N. Astratov, A.V. Barishev, A.A. Kaplyanskii, O.Z. Karimov, M.F. Limonov, Phys. Rev., E Stat. Phys. Plasmas Fluids Relat. Interdiscip. Topics $61(2000) 5784$.

[16] Y.A. Vlasov, M. Deutch, D.J. Norris, Appl. Phys. Lett. 76 (2000) 1627.

[17] A. Herzog, C.A. Paula, M.E. Darbello, F. Gallembeck, Colloid Surface A 144 (1998) 144.

[18] F. Kopnov, V. Lirtsman, D. Davidov, Synth. Met. 137 (2003) 993.

[19] E.W. Seelig, B. Tang, A. Yamilov, H. Cao, R.P.H. Chang, Mater. Chem. Phys. 80 (2003) 257.

[20] K.S. Mayya, M. Sastry, Langmuir 15 (1995) 1902.

[21] A. Stein, R.C. Schroden, Curr. Opin. Solid State. Mater. 5 (2001) 553.

[22] R.M. Amos, J.G. Rarity, P.R. Tapster, T.J. Shepherd, Phys. Rev., E Stat. Phys. Plasmas Fluids Relat. Interdiscip. Topics 61 (2000) 2959.

[23] O. Vickreva, O. Kalinina, E. Kumacheva, Adv. Mater. 12 (2000) 110.

[24] M. Yoldi, W. González-Viñas, R. Sirera, M.C. Arcos, in: M. Marciniak (Ed.), Proceedings of the 6th International Conference on Transparent Optical Networks vol. 1 (2004) 330.

[25] M. Yoldi, W. González-Viñas, M.C. Arcos, R. Sirera, J. Mater. Sci. 41 (2006) 2965.

[26] L.M. Goldenberg, J. Wagner, J. Stumpe, J. Stumpe, B.R. Paulke, E. Görnitz, Mater. Sci. Eng., C, Biomim. Mater., Sens. Syst. 22 (2002) 405.

[27] L.M. Goldenberg, J. Wagner, J. Stumpe, B.R. Paulke, E. Görnitz, Langmuir 118 (2002) 3319

[28] P. Nozar, C. Dionigi, A. Migliori, G. Calestani, L. Cademartiri, Synth. Met. 139 (2003) 667.

[29] P. Jiang, J.F. Bertone, K.S. Hwang, V.L. Colvin, Chem. Mater. 11 (1999) 2132.

[30] P. Jiang, K.S. Hwang, D.M. Mitterman, J.F. Bertone, V.L. Colvin, J. Am. Chem. Soc. 121 (1999) 11630.

[31] Y.A. Vlasov, X.-Z. Sturm, D.J. Norris, Nature 414 (2001) 289.

[32] A. Kotera, F. Furosawa, Y. Takeda, Kolloid-Z.Z. Polym. 239 (1970) 677.

[33] J.W. Goodwin, J. Hearn, C.-C. Ho, R. Ottewill, Colloid Polym. Sci. 252 (1974) 464.

[34] B.R. Paulke, P.M. Möglich, E. Knippel, A. Bude, R. Nizsche, R.H. Müller, Langmuir 11 (1995) 70.

[35] Y.Y. Lu, M.S. El-Aasser, J.W. Vanderhoff, J. Polym. Sci. Pol. Phys. 26 (1998) 1187.

[36] L. Shmuylovitch, A.Q. Shen, H.A. Stone, Langmuir 18 (2002) 3441.

[37] H.J. Schöpe, A. Barreira Fontecha, H. Hönig, J. Marqués Hueso, R. Biehl, Langmuir 22 (2000) 1828.

[38] G.S. Lazarov, N.D. Denkov, O.D. Velev, P.A. Kralchevsky, K. Nagayama, J. Chem. Soc. Faraday Trans. 90 (1994) 2077.

[39] S. Neser, C. Bechinger, P. Leiderer, T. Palberg, Phys. Rev. Lett. 79 (1997) 2348.

[40] S. Neser. PhD thesis, Universität Konstanz (1998).

[41] A.V. Petukhov, I.P. Dolbnya, D.G.A.L. Aarts, G.J. Vroege, Phys. Rev., E Stat. Phys. Plasmas Fluids Relat. Interdiscip. Topics 69 (2004) 031405.

[42] A.F.M. Barton, Handbook of Solubility Parameters, CRC Press, 1983, p. 153. 